\title{
Utilização de imagens TM/LANDSAT-5 como ferramenta no mapeamento de solos ${ }^{1}$
}

\author{
Zuleide M. C. Lima², Mateus R. Ribeiro ${ }^{3} \&$ Alexandre T. de O. Lima ${ }^{4}$
}

\begin{abstract}
1 Parte de Dissertação de Mestrado defendida pela primeira autora na UFRPE.
2 Depto. de Geologia/UFRN. Rodovia BR-101, s/n, CEP 59078-900, Natal, RN. Fone: (84) 236-2919. E-mail: zuleide@geologia.ufrn.br

${ }^{3}$ Depto. de Agronomia/UFRPE. CEP 52171-900, Recife, PE. Fone: (81) 3302-1220. E-mail: rosas@truenet.com.br (Foto)

4 Depto. Engenharia Cartográfica/UFPE. (Falecido)
\end{abstract}

Protocolo $041-17 / 4 / 2000$

\begin{abstract}
Resumo: Imagens orbitais TM/LANDSAT-5 foram tratadas por técnicas de processamento digital com o objetivo de se testar a viabilidade da utilização desta técnica em mapeamento de solos. A área estudada, parte da bacia do Rio Brígida, no sertão do Estado de Pernambuco, está delimitada pelas coordenadas UTM 428.000 a $420.000 \mathrm{mE}$, e 9.074 .000 a $9.062 .000 \mathrm{mN}$, totalizando $96 \mathrm{~km}^{2}$. Usando-se a transformação RGB das imagens do visível e do infravermelho, e a aplicação dos principais componentes associada a técnicas de realce pelos programas WFM e Álgebra do ERDAS, versão 7.4, juntamente com informações de campo, verificou-se bom desempenho do tratamento digital na delimitação dos contornos das unidades de mapeamento e na individualização de alguns desses solos. $\mathrm{O}$ tratamento digital de imagens com apoio de campo mostrou-se adequado para o mapeamento de solos no nível de levantamento de reconhecimento. As composições coloridas (RGB) das bandas do TM mais informativas, para delimitação das unidades de mapeamento de solos na área estudada, foram 1-3-5 e 4-5-7.
\end{abstract}

Palavras-chave: levantamento de solo, TM/LANDSAT-5, tratamento de imagem, ERDAS

\section{Utilization of TM/LANDSAT-5 images as a tool in soil surveys}

\begin{abstract}
Orbital imagery TM/LANSAT- 5 were treated by digital techniques aiming to evaluate the utility of this tool in soil surveys. The studied area has about $96 \mathrm{~km}^{2}$ and is part of the Brigida River basin, in the Sertão region of Pernambuco State, Brazil, limited by UTM coordinates of 428,000 to $420,000 \mathrm{mE}$ and $9,074,000$ to $9,062,000 \mathrm{mN}$. The use of RGB transformations of the visible and infrared imagery, along with WFM contrast techniques, algebraic programs of the ERDAS, version 7.4, and field informations allowed a better mapping of the soil units. The results showed that digital image processing with field support is useful for soil surveys at the reconnaissance level. The most useful colored compositions (RGB) of the TM bands, for soil mapping purposes in the studied area, were 1-3-5 and 4-5-7.
\end{abstract}

Key words: soil mapping, TM/LANDSAT, image processing, ERDAS

\section{INTRODUÇÃO}

No Brasil, país de dimensões continentais, o sensoriamento remoto constitui ferramenta indispensável para o conhecimento e o monitoramento dos recursos naturais.

Os sistemas sensores fotográficos (fotografias aéreas) também conhecidos como sensores remotos de baixa altitude, são largamente empregados nos levantamentos de solos, oferecendo ganho de tempo, precisão de limites e uma visão global da paisagem; por outro lado, as técnicas de processamento digital em imagens orbitais, embora largamente utilizadas no monitoramento do meio ambiente, como na botânica, cartografia, geologia, meteorologia e em outras áreas, ainda são pouco utilizadas pela ciência do solo.
Considerando-se que, dos recursos naturais, os solos são os que têm sido menos estudados com o uso dessas técnicas, o presente trabalho tem por objetivo avaliar a eficácia do tratamento digital de imagens do TM/LANDSAT-5, como ferramenta para o mapeamento dos solos.

\section{MATERIAL E MÉTODOS}

\section{Escolha e determinação do tamanho da área}

Os critérios para a escolha da área, foram:

- existência de um mapa de solos, executado através da metodologia tradicional;

- maior número de unidades de mapeamento de solos; 
- existência de imagens TM/LANDSAT, com o mínimo possível de nuvens e suas disponibilidades no Departamento de Engenharia Cartográfica da Universidade Federal de Pernambuco (UFPE).

O tamanho da área foi determinado em função da resolução da tela de vídeo, de 512 x 512 (Pixel) apresentando, assim, o maior elemento de resolução sem compressão ou ampliação, possibilitando, desta forma, melhor visualização da imagem.

\section{Localização e extensão da área}

A área estudada localiza-se na região da bacia do Rio Brígida, inserida na região do Projeto "Bruno não Cálcico" da EMBRAPA (1995), no sertão do Estado de Pernambuco, e está delimitada pelas coordenadas UMT 428.000 a $420.000 \mathrm{mE}$, e 9.074 .000 a $9.062 .000 \mathrm{mN}$, totalizando $96 \mathrm{~km}^{2}$. Abrange parte dos municípios de Parnamirim, Santa Maria da Boa Vista, Orocó e Cabrobó (Figura 1).

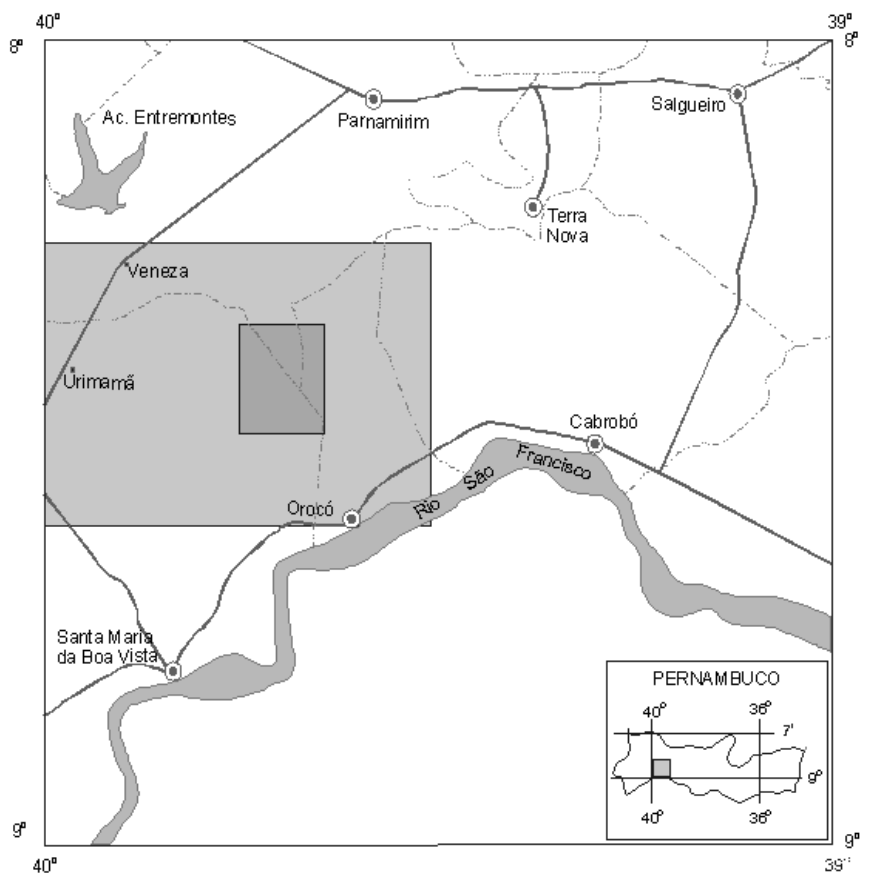

LEGENDA

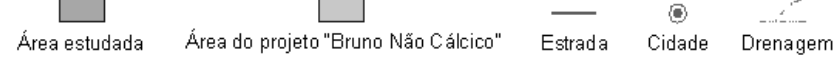
Fonte: EMBRAPA (1995)

Figura 1. Localização da área estudada

\section{Equipamento e programática}

No tratamento digital das imagens foi usado o Sistema de Análise de Dados de Recursos da Terra (Earth Resources Data Analysis System-ERDAS) na versão 7.4, lançado em 1990, nos Estados Unidos.

O ERDAS é um sistema interativo, projetado para tratar automaticamente informações multiespectrais de imagens digitais. Este sistema opera sob comando de um minicomputador que controla a execução dos algoritmos implementados, os quais permitem realçar determinados aspectos da imagem, facilitando a identificação de áreas com características espectrais semelhantes; operar transformações matemáticas nas bandas originais, gerando novas bandas transformadas; alterar a escala de trabalho etc.

As imagens TM/LANDSAT, gravadas no formato TIF, foram processadas no Adobe Photoshop (Versão 2.5) para a inclusão da legenda e impressão.

\section{Seleção de material bibliográfico e iconográfico}

O material bibliográfico incluiu trabalhos de mapeamento convencional de solos da área estudada, e trabalhos baseados nas técnicas de sensoriamento remoto.

Cartas e mapas utilizados: Folhas Topográficas Parnamirim e Santa Maria da Boa Vista-PE (SC.24-C I e SC.24-C III), na escala 1:100.000, da Diretoria do Serviço Geográfico (1982 e 1984); Mapa Exploratório - Reconhecimento de solos na escala 1:600.000, (SUDENE, 1969); e Mapas de solos do Projeto Bruno Não Cálcico, nas escalas 1:25.000 e 1:100.000 (EMBRAPA/ CODEVASF, 1995).

\section{Imagem TM/LANDSAT}

No presente trabalho foram utilizadas todas as bandas do espectro ótico do TM/Landsat-5, órbita/ponto 217/66 de 6/6/ 1992, sobre as quais foi delimitada a área de estudo.

O algoritmo "Statistics", do sistema ERDAS, foi utilizado para se calcular a matriz dos coeficientes de correlação entre as bandas e determinar as melhores combinações das três bandas estudadas neste trabalho.

As variações nas respostas espectrais dos vários alvos contidos na área de estudo foram evidenciadas por cores diferentes e suas combinações. A gradação na composição colorida representa um recurso valioso e é um procedimento rotineiro quando se pretende obter ganho de informação em determinada cena.

\section{Técnicas de realce}

As técnicas de realce usadas neste trabalho foram as seguintes: análise por principais componentes, manipulação de contraste e filtragem espacial de freqüência.

Schowengerdt apud Kuplich (1994) mostrou que imagens de diferentes bandas de uma mesma cena são visualmente similares, geralmente apresentando alta correlação, ou seja, podem apresentar informações redundantes. Esta correlação está ligada às semelhanças do padrão espectral de um alvo em faixas espectrais distintas. As transformações por Principais Componentes, permitiram reduzir ou minimizar a redundância de informações espectrais nas diferentes bandas.

Após a análise dos histogramas de freqüência dos níveis de cinza de cada banda espectral, aplicou-se a manipulação de contraste, ou seja, o nível de cinza da imagem original foi transformado em outro valor digital, de tal modo que o contraste foi aumentado. Neste processamento utilizaram-se os programas WFM e Álgebra do sistema ERDAS (Figura 2).

Uma imagem é caracterizada por diferentes níveis de cinza, que representam, em quantidades discretas, o sinal proveniente da superfície terrestre (Jensen, 1986). Com o intuito de melhorar a visualização da imagem, utilizou-se o processo de filtragem (filtro passa baixa da média, do programa IPX do ERDAS) que consiste no realce de determinados grupos de freqüência, enfatizando-se certas feições da cobertura terrestre. 
A.

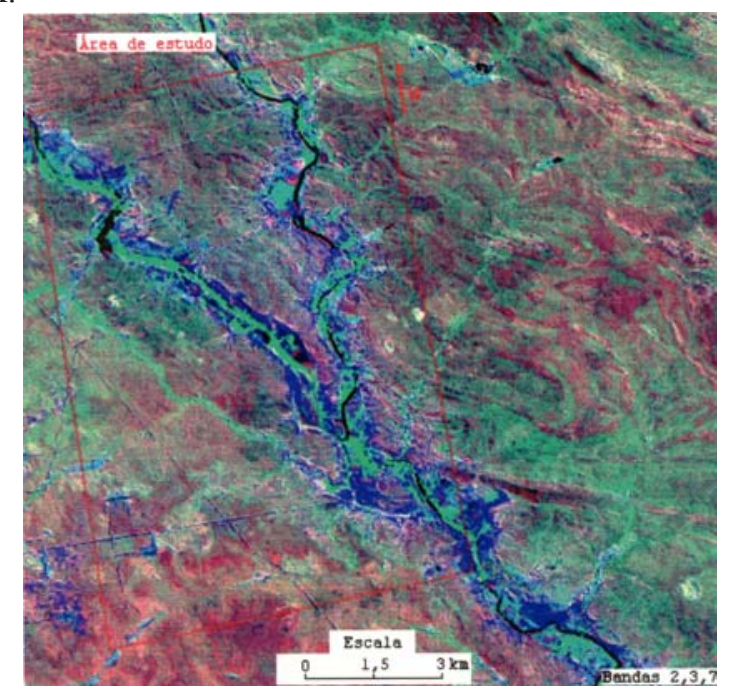

B.

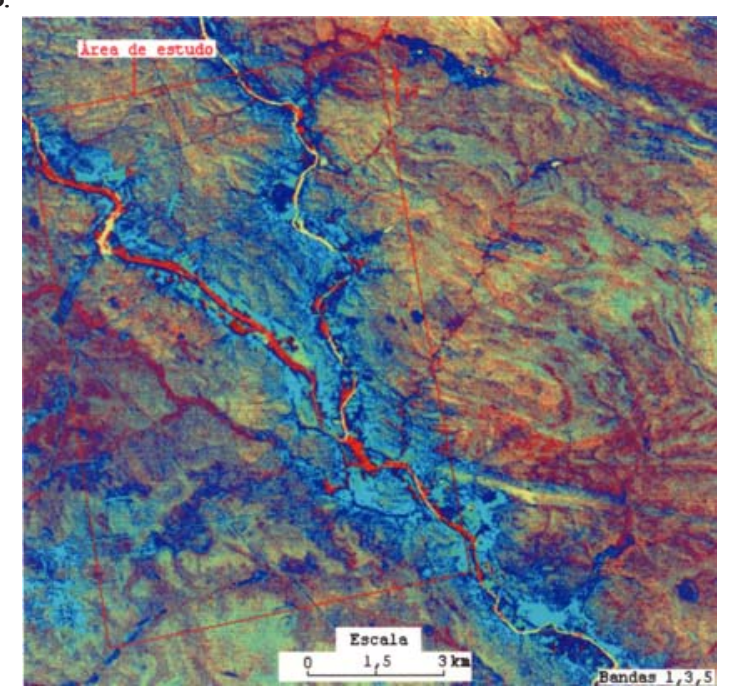

C.

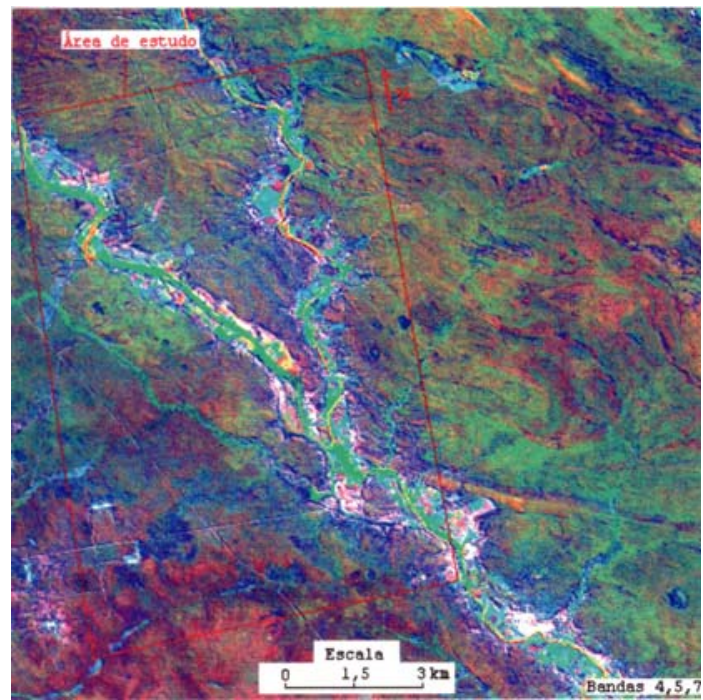

Figura 2. Transformação RGB das imagens realçadas pelos programas WFM (A e B) e Álgebra (C)

\section{Classificação automática dos padrões espectrais e complementação visual}

As técnicas de classificação digital implicam na implementação de um processo de decisão, para que o computador possa atribuir certo conjunto de pontos da imagem (pixels) a determinada classe (Novo, 1989).

O sistema ERDAS dispõe de dois tipos de algoritmo de classificação automática: classificação supervisionada e classificação não supervisionada, em que na primeira o usuário define as classes e interage com o sistema, fornecendo os dados de níveis de cinza dos alvos de interesse para cada uma dessas classes, constituindo a etapa de aquisição de amostras e treinamento. A partir das áreas de treinamento, os dados são processados pela função de densidade multivariada gausiana

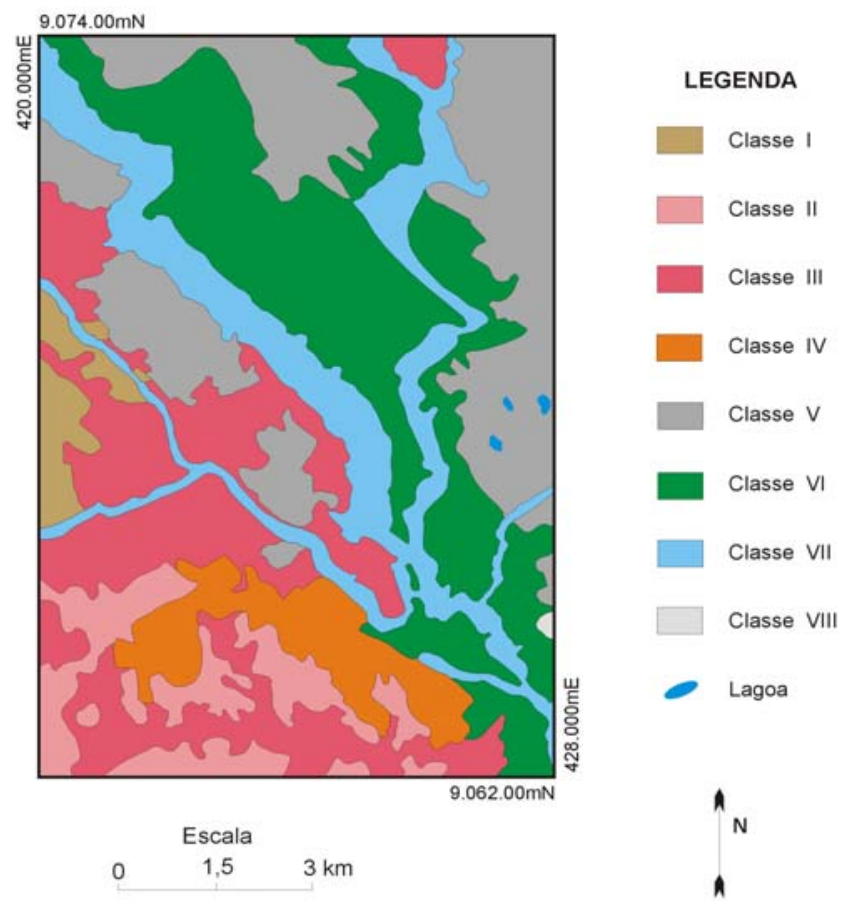

Figura 3. Classificação automática e complementação visual relacionadas às unidades de solos (imagem TM/LANDSAT-5; data 6/6/1992)

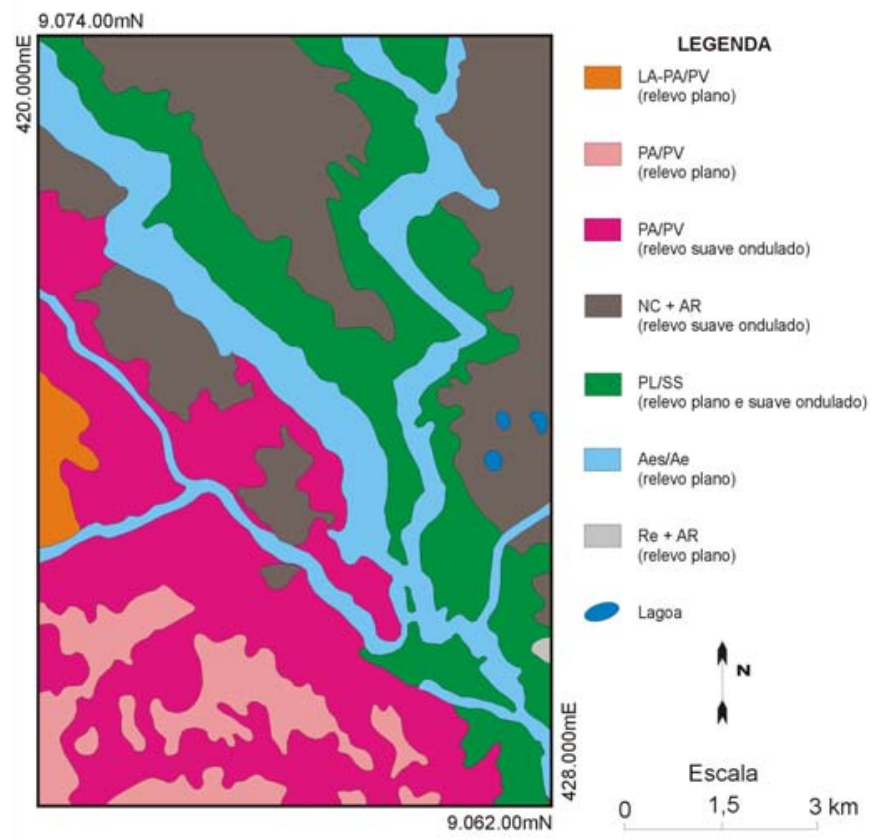

Figura 4. Mapa de reconhecimento de solos 
e agrupados nas classes definidas; já na classificação não supervisionada, o sistema executa a classificação sem a intervenção do usuário, que pode, apenas, definir o número de classes de interesse.

No uso da classificação automática não supervisionada e, posteriormente, da complementação visual, foi possível delimitar classes relacionadas a aspectos pedológicos sem, contudo, especificar os tipos de solo correspondentes a cada classe (Figura 3).

\section{Retificação geométrica}

Lima (1993) afirma que as imagens geradas por sensor remoto, tanto em plataformas aeronáuticas como em plataformas astronáuticas (tripuladas e não tripuladas) estão sujeitas a uma série de distorções espaciais de precisão cartográfica, relacionadas ao posicionamento dos objetos superficiais ou fenômenos nelas representados.

Para se eliminar as distorções, empregou-se a transformação baseada em pontos de controle no terreno (PCT) que foram identificados e posicionados de forma interativa, melhorando a precisão.

\section{Trabalhos de campo}

Durante a visita de campo percorreu-se grande parte da área de estudo através das principais vias de acesso. A carta topográfica Parnamirim SC-24-C-1 (1:100.000), o mapa de solos da EMBRAPA (1995) e o mapa preliminar da interpretação da imagem TM/LANDSAT-5, serviram como referência para o reconhecimento das unidades de mapeamento de solos.

A identificação dos solos foi realizada apenas pela observação dos cortes das estradas e tradagens, não sendo feita nenhuma descrição ou coleta de amostras. A classificação baseou-se nas classes de solo, mapeadas no Levantamento da EMBRAPA, cujos limites foram atualizados, individualizandose os componentes de algumas associações.

Algumas manchas de solo foram definidas somente em função do padrão espectral dominante, pois o reduzido tempo de campo não permitiu a observação de todas elas.

Os pontos escolhidos para o estudo de campo tiveram suas coordenadas determinadas nas cartas topográficas e a localização deles, no campo, foi feita pelo posicionador Garmin 38, que utiliza o sistema GPS na determinação de coordenadas.

\section{RESULTADOS E DISCUSSÃO}

O mapa de reconhecimento de solos (Figura 4) reflete os resultados da integração dos dados da classificação automática e complementação visual, com os dados de campo. A legenda foi elaborada a partir das unidades de mapeamento da EMBRAPA (1995) com algumas modificações decorrentes das observações de campo e da individualização de algumas classes pela metodologia testada neste trabalho. A classificação dos solos foi aproveitada integralmente, pois não foi objetivo do presente trabalho realizar nenhum tipo de coleta ou análise dos solos.

As unidades de mapeamento individualizadas estão descritas a seguir, de forma sucinta, porém se enfatizando os aspectos que influenciaram na sua separação.
Associação de Latossolo Amarelo e Podzólico Amarelo/ Vermelho Amarelo de relevo plano (LA+PA/PV)

Estes solos são geralmente profundos, permitindo boa retenção de umidade e ocorrem normalmente em relevo plano, nos topos aplainados dos Tabuleiros interioranos.

Oliveira et al. (1992) confirmam que os Latossolos Amarelos se assemelham aos Podzólicos Amarelos. A diferença entre eles é sutil, sendo necessário a descrição de perfis e em alguns casos, a análise física.

Para delimitação desta unidade de mapeamento, empregaram-se as bandas 5 e 7 que, segundo Novo (1989) têm, como principais aplicações, a determinação de umidade dos solos e da vegetação. As composições coloridas, associadas a técnicas de realce, que melhor responderam, neste caso, foram TM 1R,3G,5B e TM 4R,5G,7B, fortalecendo os resultados do trabalho apresentado por Nanni \& Rocha (1995).

\section{Associações de Podzólico Amarelo e Vermelho Amarelo de relevo plano e suave ondulado (PA/PV)}

Compreendem solos de propriedades heterogêneas, de drenagem boa a moderada e variam de muito profundos a rasos (EMBRAPA, 1995). Ocupam situações topográficas com relevo plano e suave ondulado, respectivamente. Os PA/PV relevo plano, encontram-se nos topos dos Tabuleiros; já os de relevo suave ondulado ocorrem nas encostas e nos Tabuleiros dissecados, em áreas com sedimentos pouco espessos sobre rochas diversas.

As bandas 5 e 7 destacaram as diferenças topográficas entre as duas unidades de mapeamento, e as composições coloridas TM 1R,3G,5B e TM 4R,5G,7B, associadas às técnicas de realce, ressaltaram os aspectos do relevo. Estas composições espectrais também foram utilizadas com sucesso por Nanni \& Rocha (1995) e por Ghani et al. (1995).

\section{Associação de Bruno Não Cálcico e Solos Litólicos de relevo suave ondulado $(\mathrm{NC}+\mathrm{Re})$}

Os Brunos Não Cálcicos predominam nesta unidade de mapeamento. São solos pouco profundos ou rasos, com grande pedregosidade superficial, constituída predominantemente por cascalhos, calhaus e matacões de quartzo que, na maioria das vezes, se encontram distribuídos apenas na superfície do solo.

Em virtude de ocorrerem em regiões muito secas, com prolongados períodos de estiagem, a vegetação original é do tipo caatinga arbustiva aberta, cobertura pouco efetiva no provimento de matéria orgânica naturalmente adicionada ou incorporada ao solo, resultando no desenvolvimento de um horizonte A, de pouca espessura, geralmente claro, com teor de carbono relativamente baixo.

As bandas 4 e 5 revelaram resultados satisfatórios no tocante às diferenças entre os Brunos Não Cálcicos e as demais unidades de mapeamento, confirmando os resultados dos trabalhos de Moraes et al. (1995) e Oliveira (1995) que utilizaram essas mesmas bandas para maior distinção das diferentes unidades de mapeamento de solo. As composições coloridas mais informativas foram TM 1R, 3G, 5B, TM 3R, 4G, 5B e TM 4R, 5G, 7B. 


\section{Associação de Planossolos e Solonetz Solodizado (PL/SS)}

Trata-se de solos de drenagem imperfeita, profundidade efetiva geralmente pequena, com ou sem pedregosidade superficial. Nas condições de clima semi-árido, pela morfologia fica muito difícil a distinção desses solos; entretanto, dados disponíveis indicam que na área estudada predominam os Planossolos (EMBRAPA, 1995).

Tendo em vista a necessidade de maximização do contraste entre superfície líquida e superfície desnuda de relevo plano, utilizaram-se as bandas 4 e 5 para gerar as composições coloridas TM 1R, 3G, 5B e TM 4R, 5G, 7B, que permitiram, através de técnicas de realce, a melhor eficiência na interpretação desta unidade de mapeamento, corroborando com o trabalho de Oliveira (1995), cujas bandas mais informativas para detecção de relevo, textura e drenagem, também foram as bandas 4 e 5 do TM.

\section{Associação de Solos Aluviais (AeS/Ae)}

Por definição, esses solos se desenvolvem apenas nas planícies aluvionais, em depósitos recentes de origem fluvial. $\mathrm{Na}$ zona semi-árida do Nordeste, os Solos Aluviais, por oferecerem melhor condição de umidade, são dos mais importantes para a região, não só pelo uso com diversas culturas sob irrigação como, também, pelas culturas de sequeiro, como milho, feijão e algodão. É importante ressaltar que esses solos, pelos terrenos que ocupam, estão sujeitos a riscos de inundação.

A ocorrência desta unidade de mapeamento encontra-se restrita às margens de cursos d'água, formando faixas mais ou menos estreitas e, algumas vezes, descontínuas.

As bandas 2, 4 e 7 foram as que melhor responderam à detecção dos Solos Aluviais ou Aluvio-Coluviais; isto se deve à reflectância de vegetação verde sadia, proporcionada pela banda 2, e pela boa delimitação de corpos d'água que as bandas 4 e 7 possibilitam, concordando com as proposições de Garcia (1982) e Novo (1989). As melhores composições coloridas foram TM 2R, 3G, 7B, TM 3R, 4G, 5B e TM 4R, 5G, 7B.

\section{Associação de Solos Litólicos e afloramentos de rocha ( $R e+A R)$}

São solos rasos, pouco evoluídos, com horizonte superficial A assente diretamente sobre a camada R. Ocupam várias situações topográficas, sendo comuns nas áreas movimentadas, serras e serrotes, encostas de vales e, menos freqüentemente, em áreas aplainadas, e se originam de rochas diversas, como gnaisses, granitos, micaxistos e quartzitos, entre outras.

Verificou-se que as melhores composições foram as formadas pelas bandas 5 e 7. Segundo Bärhr (1991) a banda 7 é a que se adapta especialmente a estudos de rochas. As composições coloridas que melhor discriminaram esta unidade de mapeamento, foram TM 3R, 4G, 5B e TM 4R, 5G, 7B, as quais permitiram associar-se as feições das imagens aos solos da área de estudo, sendo que a composição TM 4R, 5G, 7B foi a mais informativa.

\section{CONCLUSÕES}

1. Os resultados do tratamento digital de imagens, associados a trabalho de campo, mostraram-se adequados para o mapeamento de solos a nível de levantamento de reconhecimento, na escala 1:100.000.

2. A composição colorida TM 1R, 3G, 5B que combina bandas na faixa espectral do visível e infravermelho refletido, submetida às técnicas de realce WFM, ofereceu melhores recursos visuais na individualização dos Latossolos, Brunos Não-Cálcicos e Planossolos.

3. Para diferenciação entre os Podzólicos Vermelho-Amarelos de relevo plano e os de relevo suave ondulado, as composições coloridas mais adequadas foram TM 1R, 3G, 5B e TM 4R, 5G, 7B.

4. Os Solos Aluviais ou Colúvio-Aluviais que ocorrem associados à rede de drenagem, foram melhor individualizados pela composição colorida TM 4R, 5G, 7B. Esta mesma composição serviu para delimitação dos Solos Litólicos.

5. A classificação automática de padrões e a complementação visual das imagens TM/LANDSAT-5, mostraram-se mais eficientes para a individualização das diferentes classes de solo, quando foram associadas à observação de elementos fotopedológicos como drenagem, relevo e textura da imagem.

6. A continuidade das pesquisas nesta linha vai permitir a operacionalidade da metodologia, visando maior rapidez na obtenção dos dados para mapeamento e aumento no grau de confiabilidade das informações obtidas através de dados por satélites.

\section{LITERATURA CITADA}

Bähar, H.P. Processamiento digital de imágens: Aplicaciones fotogramétrie y teledeteccion. Eschborn: Deustsche Gesells Chaft fur Technische, 1991.p. 259-316: Sistemas actuales y futuros para aquisisicion de dados en teledeteccion y fotogrametrie.

EMBRAPA. - Empresa Brasileira de Pesquisa Agropecuária. Projeto Bruno Não Cálcico: Levantamento semidetalhado de solos de uma área piloto da zona semi-árida do nordeste. Recife: EMBRAPA/CNPS, 1995.30p.

Garcia, C.J. Sensoriamento remoto: princípios e interpretação de imagens. São Paulo: Nobel, 1982. ?p.

Ghani, N.L.B.; Rocha, H.O. da; Scmidlin, D.; Nanni, M.R.; Rocha, C.H. Levantamento de solo do Parque Estadual Pico Marumbi - Serra do Mar, PR. In: Congresso Brasileiro de Ciência do Solo, 25, 1995, Viçosa, MG. Anais... Viçosa, MG. SBCS, 1995.p.1643-1645.

Kuplich, T.M. Estudo da complementaridade de imagens óticas (LANDSAT/TM) e de RADAR (ERS-1/SAR) na discriminação temática de uso da terra. São José dos Campos: INPE. 1994. 109p. Dissertação Mestrado

Jensen, J.R. Introductory digital image processing: Remote sensing perspective. New Jersey: Prentice-Hall, 1986. 179p.

Lima, A.T. de O. Aplicação de imagens satelitais no mapeamento de aspectos geológicos. Recife: UFPE, 1993, 67p. Dissertação Mestrado

Morais, J.F.L.; Volkoff, B.; Cerri, C.C. Uso de imagens digitais LANDSAT-TM no levantamento de áreas ocupadas com pastagens em Rondônia. In: Congresso Brasileiro de Ciência do Solo, 25, 1995, Viçosa, MG. Anais... Viçosa, MG: SBCS, 1995.p.1454-1456. 
Nanni, M.R.; Rocha, H.O. da. Delimitação de unidades fisiográficas para estudo de solos através da associação da análise de agrupamentos e técnicas de geoprocessamento e sensoriamento remoto. In: Congresso Brasileiro de Ciência do Solo, 25, 1995, Viçosa, MG. Anais... Viçosa, MG: SBCS, 1995. p.1651-1653.

Novo, E.M.L. de M. Sensoriamento remoto: princípios e aplicações. São Paulo: Edgard Blucher, 1989, 307p.
Oliveira, J.B. de; Jacomine, P.K.T.; Camargo, M.N. Classes gerais de solos do Brasil: guia auxiliar para seu reconhecimento. Jaboticabal: Funep, 1992. 201p.

Oliveira, S.B.P. de. Imagens TM-LANDSAT para levantamentos pedológicos nas condições semi-áridas dos sertões dos Inhamuns - Salgado, Ceará. In: Congresso Brasileiro de Ciência do Solo, 25, 1995, Viçosa, MG. Anais... Viçosa, MG: SBCS, 1995. p.1698-1699. 